\title{
Safer UAV Piloting: A Robust Sense-and-Avoid Solution for Remotely Piloted Quadrotor UAVs in Complex Environments
}

\author{
Min Wang ${ }^{1}$ Holger $\operatorname{Voos}^{1}$
}

\begin{abstract}
Current commercial UAVs are to a large extent remotely piloted by amateur human pilots. Due to lack of teleoperation experience or skills, they often drive the UAVs into collision. Therefore, in order to ensure safety of the UAV as well as its surroundings, it is necessary for the UAV to boast the capability of detecting emergency situation and acting on its own when facing imminent threat. However, the majority of UAVs currently available in the market are not equipped with such capability. To fill in the gap, in this paper we present a complete sense-and-avoid solution for assisting unskilled pilots in ensuring a safe flight. Particularly, we propose a novel nonlinear vehicle control system which takes into account of sensor characteristics, an emergency evaluation policy and a novel optimization-based avoidance control strategy. The effectiveness of the proposed approach is demonstrated and validated in simulation with multiple moving objects.
\end{abstract}

\section{INTRODUCTION}

Multirotor Unmanned Aerial Vehicles (UAVs), especially quadrotor UAVs, have been the focus of many research works in recent years, thanks to their increasing availability in the market, and their agility and maneuvoerability through complex environments. They have been employed in a wide range of applicatoin scenarios, such as search and rescue, surveillance and inspection, forestry and precision farming ect. In all these applications, collision avoidance is a top priority during missions, so as to ensure safety of both the UAV and its surrounding environment. Current commercial UAVs are to a large extent remotely piloted by human pilots. The human pilot (or operator), however, due to lack of operating skills or limited situation awareness, often times fails to avoid collisions. Therefore, it is necessary to have an onboard sense-and-avoid solution as an emergency safety assistance system, which is able to identify imminent collision threat and override remote pilot's commands when necessary in order to ensure safety.

In the literature, there have been many research works focusing on sense-and-avoid solution (or collision avoidance solution) for autonomous UAVs [3] [4] [5], while only very few works focus on that for remotely piloted UAVs [6]. Collision avoidance system for autonomous UAVs are goal-oriented, while for remotely piloted UAVs there is no goal location to reach after the avoidance manoeuvre, which leaves more flexibility in designing the avoidance strategy. Some works propose avoidance techniques which try to preserve as much as possible the intent of the human pilot.

*This work was supported by the "Fonds National de la Recherche" (FNR), Luxembourg, under the project C15/15/10484117 (BEST-RPAS).

${ }^{1}$ Automation and Robotics Research Group, Interdisciplinary Centre for Security, Reliability and Trust (SnT), University of Luxembourg, Luxembourg $\{$ min.wang, holger.voos\} duni.lu
However, except for certain specific applications, it is rarely necessary to preserve the intent of the pilot as the majority of amateur pilots drive UAVs into collision situations without any intent of doing so but rather due to lack of skills.
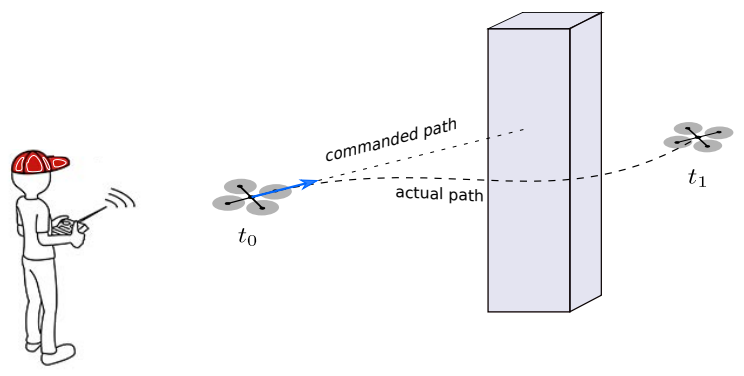

Fig. 1. Collision Avoidance of Remotely Piloted UAV

The main contribution of this paper is that we propose a complete 2D LIDAR-based sense-and-avoid solution for assisting unskilled pilot in ensuring safe flight. Within this overall solution framework, we propose a novel nonlinear vehicle controller adapted to the characteristics of LIDAR sensing, an emergency evaluation policy and a novel avoidance control strategy which takes inspiration from missile guidance. The effectiveness of the proposed approach is validated with a two-moving-objects scenario in simulation.

\section{RELATED WORK}

Different sense-and-avoid solutions have been proposed in the literature with varying sensing modalities and avoidance techniques, mostly for autonomous UAVs. [3] presents a collision avoidance algorithm which only utilizes measuremnets from a gimbaled monocular camera. Line-of-Sight (LOS) angle and time-to-collision (TTC) are abstracted through computer vision algorithms, which are subsequently used to form an avoidance control law controlling the angular rate of the UAV. Simulation is conducted in a single-moving-object scenario and a pure-static-objects scenario to validate the proposed avoidance control law. In [4] a goal-directed 3D reactive obstacle avoidance algorithm is proposed for rotorcraft UAVs equipped with a stereo camera pair and a 2D LIDAR sensor. 3D occupancy map is used as world representation, where the avoidance algorithm projects a cylindrical safety volume ahead of the UAV to detect potential collisions. Once potential collision is detected, an expanding elliptical search is carried out to find an escape point. Both simulation and real flight experiments have been conducted to validate the algorithm in static environments with trees and communication towers. [5] introduces a reactive guidance strategy for 
collision avoidance, which uses bearing-only measurements provided by a camera. The guidance law is derived from sliding mode control theory, and it moves an obstacle in the sensor field-of-view to a desired constant bearing angle, which leads the UAV to maintain a constant distance from the obstacle. The proposed guidance law can be used to avoid collision into circular obstacles and to follow straight and curved walls at a safe distance.

Very few works focus on automatic collision avoidance for pilot assistance system. [6] presents an automatic collision avoidance approach for assisting human operation, so that the pilot can focus on global mission objectives. The proposed control method is essentially a model predictive control approach. In case an imminent collision is foreseen, the operator's command is overriden with the nearest control input that avoids the collision while preserving the operator's intent as closely as possible. The mechanisms of sensing, threat evaluation, and interaction between human operator and UAV are not discussed and assumed to be working. Experiment is performed in a static lab environment, with the assumption of full environment awareness.

Most of the existing works on sense-and-avoid systems are vision-based. Additionally, the proposed algorithms in most works are validated in simulation or experiments with either a single-moving obstacle or a pure static environment. Their capabilities of avoidance in complex environments where there exist multiple moving objects, are barely investigated.

\section{Dynamic Model Of Quadrotor}

The general dynamic model of a quadrotor has been derived in numerous papers and thus is only briefly introduced here, while further details can be found in e.g. [7], [8], [9] and [10]. We adopt three major reference frames for describing the dynamic model of a quadrotor: inertial frame, vehicle frame and body frame, as shown in Fig. 2. The inertial frame is defined in the standard NED (North, East, Down) form. The origin of the vehicle frame is fixed on the $\mathrm{UAV}$, vehicle frame is a mere translation of the inertial frame. The body frame is also fixed on the UAV, but it rotates with the UAV with regard to the vehicle frame. The dynamics of a quadrotor can be described by the external forces and torques exerted on the center of mass of the vehicle as follows:

$$
\begin{gathered}
\ddot{\boldsymbol{p}}^{i}=g \cdot\left(\begin{array}{c}
0 \\
0 \\
1
\end{array}\right)-\boldsymbol{R}_{b}^{i}\left(\boldsymbol{\Omega}^{v}\right) \cdot b / m \sum_{i=1}^{4} \omega_{i}^{2} \cdot\left(\begin{array}{l}
0 \\
0 \\
1
\end{array}\right) \\
\boldsymbol{I} \ddot{\boldsymbol{\Omega}}^{b}=-\left(\dot{\boldsymbol{\Omega}}^{b} \times \boldsymbol{I} \dot{\boldsymbol{\Omega}}^{b}\right)-\boldsymbol{M}_{G}^{b}+\boldsymbol{M}^{b}
\end{gathered}
$$

where $\boldsymbol{p}^{i}=(x, y, z)^{T}$ is the position of the vehicle in the inertial frame. $\boldsymbol{\Omega}^{v}=(\phi, \theta, \psi)^{T}$ describes the orientation of UAV in the vehicle frame expressed with roll, pitch and yaw angles. $\boldsymbol{\Omega}^{b}=\left(\phi^{b}, \theta^{b}, \psi^{b}\right)^{T}$ is the Euler angles expressed in the body frame. $\boldsymbol{R}_{b}^{i}\left(\Omega^{v}\right)$ is the rotation matrix for transforming from body frame to inertial frame. $g$ is the gravitational acceleration. $b$ is the thrust factor. $m$ is the mass of the UAV. $\omega_{i}$ is the speed of rotor $i . I$ is the inertia matrix with diagonal elements $I_{x}, I_{y}$ and $I_{z} . M_{G}^{b}$ is the gyroscopic torque. $\boldsymbol{M}^{b}$ describes the torques applied to vehicle.

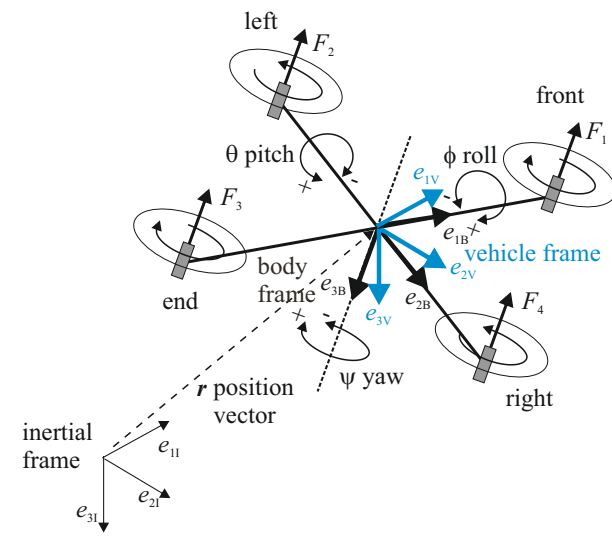

Fig. 2. Reference Frame System of Quadrotor

If we assume that the quadrotor flies in a way that is not too far away from the hovering state, thus the angles $\phi$ and $\theta$ are small, then following approximation is valid:

$$
\left(\dot{\phi}^{b}, \dot{\theta}^{b}, \dot{\psi}^{b}\right)^{T} \approx(\dot{\phi}, \dot{\theta}, \dot{\psi})^{T}
$$

We define $\boldsymbol{x}=\left(x^{i}, y^{i}, z^{i}, \dot{x}^{i}, \dot{y}^{i}, \dot{z}^{i}, \phi, \theta, \psi, \dot{\phi}^{b}, \dot{\theta}^{b}, \dot{\psi}^{b}\right)^{T}$ as the state vector, and evaluation of Eq. (1) - (5) yields following state dynamics model:

$$
\dot{\boldsymbol{x}}=\left(\begin{array}{c}
x_{4} \\
x_{5} \\
x_{6} \\
-\left(\cos x_{7} \sin x_{8} \cos x_{9}+\sin x_{7} \sin x_{9}\right) \frac{u_{1}}{m} \\
-\left(\cos x_{7} \sin x_{8} \sin x_{9}-\sin x_{7} \cos x_{9}\right) \frac{u_{1}}{m} \\
g-\left(\cos x_{7} \cos x_{8}\right) \frac{u_{1}}{m} \\
x_{10} \\
x_{11} \\
x_{12} \\
x_{11} x_{12} I_{1}-\frac{I_{R}}{I_{x}} x_{11} g(\boldsymbol{u})+\frac{L}{I_{x}} u_{2} \\
x_{10} x_{12} I_{2}+\frac{I_{R}}{I_{y}} x_{10} g(\boldsymbol{u})+\frac{L}{I_{y}} u_{3} \\
x_{10} x_{11} I_{3}+\frac{1}{I_{z}} u_{4}
\end{array}\right)
$$

where

$$
\begin{aligned}
& u_{1}=b\left(\omega_{1}^{2}+\omega_{2}^{2}+\omega_{3}^{2}+\omega_{4}^{2}\right) \\
& u_{2}=b\left(\omega_{2}^{2}-\omega_{4}^{2}\right) \\
& u_{3}=b\left(\omega_{1}^{2}-\omega_{3}^{2}\right) \\
& u_{4}=d\left(\omega_{1}^{2}+\omega_{3}^{2}-\omega_{2}^{2}-\omega_{4}^{2}\right)
\end{aligned}
$$

where $d$ is the drag factor. $\boldsymbol{u}=\left(u_{1}, u_{2}, u_{3}, u_{4}\right)^{T}, g(\boldsymbol{u})=$ $\omega_{1}-\omega_{2}+\omega_{3}-\omega_{4}, I_{1}=\left(I_{y}-I_{z}\right) / I_{x}, I_{2}=\left(I_{z}-I_{x}\right) / I_{y}$, $I_{3}=\left(I_{x}-I_{y}\right) / I_{z}, I_{R}$ is the rotor inertia and $L$ is the length of the lever.

\section{Vehicle Controller Design}

Our vehicle controller is desgined in such a way that it takes into account the characteristics of the sensing unit, and thus supports effective sensing. We employ a 2D LIDAR (e.g. Hokuyo UTM-30LX) in our sense-and-avoid solution, which provides a $270^{\circ}$ horizontal field of view (FOV). It 
is assumed that during the flight the UAV maintains a nearhovering state, which ensures that the LIDAR can effectively sense the surrounding of the UAV. Though quadrotor UAV is capable of flying in the $x^{b}$ and $y^{b}$ direction without yawing, this way of flying is not desirable for our solution as when the UAV flies sideways, the FOV of the sensing unit does not provide full coverage of the sides.

Therefore, in this section we propose a nonlinear vehicle control system which realizes a forward-facing flight behavior in the $x-y$-plane, while maintaining a desired altitude in $z$-direction. It means that the front arm of the quadrotor as defined in Fig. 2 must always point in the direction of the desired velocity vector in the $x$-y-plane in order to be able to always sense in the flight direction. The flight control consists of two main components, an inner attitude control loop that tries to achieve a desired orientation as fast as possible and an outer velocity control loop that realizes the desired velocity vector as well as the forward-facing flight.

\section{A. Attitude Control System}

The attitude control system is based on own previous work [10] and will therefore only be described very briefly here. The attitude control considers the last six ordinary differential equations (ODEs) of Eq. (4), which describe the dynamics of Euler angles and angular rates of the quadrotor. Euler angles can be obtained by pure integration. For the submodel formed by the last three ODEs, we firstly simplify the model by neglecting the gyroscopic terms as follows:

$$
\left(\begin{array}{l}
\dot{x}_{10} \\
\dot{x}_{11} \\
\dot{x}_{12}
\end{array}\right)=\left(\begin{array}{l}
x_{11} x_{12} I_{1}+\frac{L}{I_{x}} u_{2} \\
x_{10} x_{12} I_{2}+\frac{L}{I_{y}} u_{3} \\
x_{10} x_{11} I_{3}+\frac{1}{I_{z}} u_{4}
\end{array}\right)
$$

and then apply feedback linearization technique in order to obtain a linear system:

$$
\begin{aligned}
& u_{2}=f_{2}\left(x_{10}, x_{11}, x_{12}\right)+u_{2}^{*} \\
& u_{3}=f_{3}\left(x_{10}, x_{11}, x_{12}\right)+u_{3}^{*} \\
& u_{4}=f_{4}\left(x_{10}, x_{11}, x_{12}\right)+u_{4}^{*}
\end{aligned}
$$

where $u_{2}^{*}, u_{3}^{*}, u_{4}^{*}$ are introduced new input variables. We define

$$
\begin{aligned}
& f_{2}\left(x_{10}, x_{11}, x_{12}\right)=\frac{I_{x}}{L}\left(K_{2} x_{10}-x_{11} x_{12} I_{1}\right) \\
& f_{3}\left(x_{10}, x_{11}, x_{12}\right)=\frac{I_{y}}{L}\left(K_{3} x_{11}-x_{10} x_{12} I_{2}\right) \\
& f_{4}\left(x_{10}, x_{11}, x_{12}\right)=I_{z}\left(K_{4} x_{12}-x_{10} x_{11} I_{3}\right)
\end{aligned}
$$

with $K_{2}, K_{3}, K_{4}$ being constant paramenters with negative real values. With Eq. (7) - (8), Eq. (6) can be transferred into a set of linear and decoupled ODEs. Details about the dynamics and stability of this subcontrol system can be found in [10]. If we define $u_{2}^{*}=w_{2}\left(x_{7 d}-x_{7}\right), u_{3}^{*}=w_{3}\left(x_{8 d}-\right.$ $\left.x_{8}\right), u_{4}^{*}=w_{4}\left(x_{9 d}-x_{9}\right)$, where $x_{7 d}, x_{8 d}$ and $x_{9 d}$ are desired Euler angles generated from velocity control system, together with the current state vector of the UAV, we can ultimately obtain the overall control input for UAV $u_{2}, u_{3}$ and $u_{4}$.

\section{B. Velocity Control System}

The command to the vehicle control system is from the human pilot, a desired velocity vector in the $x$ - $y$-plane given by $v_{x d}=x_{4 d}, v_{y d}=x_{5 d}$, and a desired altitude $z_{d}=x_{3 d}$ in $z$-direction.

In order to obtain the desired Euler angles for the inner control loop and the UAV overall control input $u_{1}$, we consider follwing three equations from Eq. (4):

$$
\begin{aligned}
& \dot{x}_{4}=-\left(\cos x_{7 d} \sin x_{8 d} \cos x_{9 d}+\sin x_{7 d} \sin x_{9 d}\right) \frac{u_{1}}{m} \\
& \dot{x}_{5}=-\left(\cos x_{7 d} \sin x_{8 d} \sin x_{9 d}-\sin x_{7 d} \cos x_{9 d}\right) \frac{u_{1}}{m} \\
& \dot{x}_{6}=g-\left(\cos x_{7 d} \cos x_{8 d}\right) \frac{u_{1}}{m}
\end{aligned}
$$

Substitute the variables as follows

$$
\left(\begin{array}{c}
\dot{x}_{4} \\
\dot{x}_{5} \\
\dot{x}_{6}
\end{array}\right)=\boldsymbol{f}\left(x_{7 d}, x_{8 d}, x_{9 d}, u_{1}\right)=\left(\begin{array}{c}
\tilde{u}_{1} \\
\tilde{u}_{2} \\
\tilde{u}_{3}
\end{array}\right)
$$

where

$$
\begin{aligned}
& \tilde{u}_{1}=k_{1}\left(x_{4 d}-x_{4}\right) \\
& \tilde{u}_{2}=k_{2}\left(x_{5 d}-x_{5}\right) \\
& \tilde{u}_{3}=k_{3}\left(x_{3 d}-x_{3}\right)-k_{4} x_{6}
\end{aligned}
$$

With this substitution, we essentially form a closed loop control of the UAV velocities, and apply P-controllers to $x$ $y$-plane velocities, and a PD-controller for altitude control. From Eq. (9) - (11) we can then obtain $x_{7 d}, x_{8 d}, x_{9 d}$ and $u_{1}$. Since we would like to maintain a forward-facing flight, the desired yaw angle is required to be as

$$
x_{9 d}=\arctan \left(\frac{x_{5 d}}{x_{4 d}}\right)
$$

As we assume that the UAV flies near hovering state, the desired roll and pitch angles are then sufficiently small, which allows small angle approximation:

$$
\begin{aligned}
& \sin x_{7 d} \approx x_{7 d}, \cos x_{7 d} \approx 1 \\
& \sin x_{8 d} \approx x_{8 d}, \cos x_{8 d} \approx 1
\end{aligned}
$$

If we define

$$
\cos x_{9 d}=\alpha, \sin x_{9 d}=\beta
$$

and then insert Eq. (13) and (14) into Eq.(9), it yields

$$
\begin{aligned}
& \tilde{u}_{1}=-\left(\alpha x_{8 d}+\beta x_{7 d}\right) u_{1} / m \\
& \tilde{u}_{2}=-\left(\beta x_{8 d}-\alpha x_{7 d}\right) u_{1} / m \\
& \tilde{u}_{3}=g-u_{1} / m
\end{aligned}
$$

Using the last equation of (15) allows the calculation of the unknown input $u_{1}$ as

$$
u_{1}=m\left(g-\tilde{u}_{1}\right)
$$

Since $u_{1}$ is obtained, the first two equations of (15) become linear equations that can be solved as

$$
\left(\begin{array}{l}
x_{7 d} \\
x_{8 d}
\end{array}\right)=\left(\begin{array}{cc}
-\frac{u_{1}}{m} \beta & -\frac{u_{1}}{m} \alpha \\
\frac{u_{1}}{m} \alpha & -\frac{u_{1}}{m} \beta
\end{array}\right)^{-1} \cdot\left(\begin{array}{c}
\tilde{u}_{1} \\
\tilde{u}_{2}
\end{array}\right)
$$




\section{Collision Avoidance System}

Our collision avoidance system consists of three major functional modules, namely, sensing module, emergency evaluation module and collision avoidance module, as shown in Fig. 3. In the rest of this section, we will introduce them in detail.

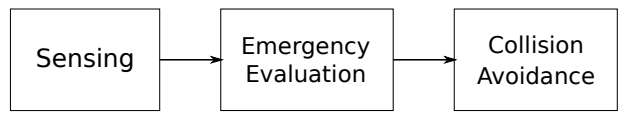

Fig. 3. Functional Diagram of Collision Avoidance System

\section{A. Sensing and Emergency Evaluation}

The sensing module contains object detection and tracking functionalities based on a 2D LIDAR, where objects in the environment are represented as cylinders with infinite height (viewed as circles in 2D horizontal plane), and a Kalman Filter is applied to track the position of objects and estimate their velocities. For detailed explanation, please refer to our previous work in [11].

Though the LIDAR we currently employ has only $270^{\circ}$ planar field of view, since the UAV is forward moving, i.e. the UAV is confined to move only in the direction where the LIDAR is front facing, and the sensing module memorizes the detected surrroudings for a short period of time, we can reasonably predict that there would be no object in the blind zone of LIDAR, with the assumption that there are no object actively pursuing the UAV and aiming to hit it from behind.

Since we would like to assess the situation around the UAV in order to decide if there is imminent threat and if the UAV should react to resolve the emergency, it would be a good idea to divide the area around the UAV into regions of different safety levels. Similar idea appeared in [12], in this paper we propose a modified definition of safety zone set.

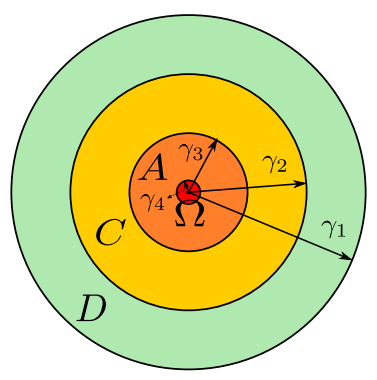

Fig. 4. Defined Safety Zones around UAV for Emergency Evaluation

The nearest area around the UAV is defined as Anti-object Zone $\Omega$, as indicated as red circular region in Fig. 4. This is the most safety-critical zone where no object should enter, otherwise collision with the UAV would take place.

We define an integer set $\mathbb{Z}=\{1,2, \ldots, n\}$ with $n$ being the number of detected objects in the environment, and define $\boldsymbol{P}_{Q}$ and $\boldsymbol{P}_{p_{i}}$ as the 2D positions of the UAV and the $i$ th object (since in this paper we assume that the UAV keeps flight at a certain height level, we can then focus on $x$-y-plane), i.e.
$\boldsymbol{P}_{Q}, \boldsymbol{P}_{p_{i}} \in \mathbb{R}^{2}$. The radius of the $i$ th object is defined as $\lambda_{i}$. Anti-object Zone $\Omega$ is then defined as follows:

$$
\boldsymbol{\Omega}=\left\{\left\|\boldsymbol{P}_{Q}-\boldsymbol{P}_{p_{i}}\right\|-\lambda_{i} \leq \gamma_{4}, i \in \mathbb{Z}\right\}
$$

where $\gamma_{4}$ denotes the required minimum separation distance between the center of the UAV and the periphery of an object in order to ensure safety.

The next level of safety zone is defined as Avoidance Zone, $\boldsymbol{A}$, as indicated in orange in Fig. 4. If there is object within this region, then imminent threat for the UAV is considered present, and action must be taken to avoid collision.

$$
\boldsymbol{A}=\left\{\gamma_{4} \leq\left\|\boldsymbol{P}_{Q}-\boldsymbol{P}_{p_{i}}\right\|-\lambda_{i} \leq \gamma_{3}, i \in \mathbb{Z}\right\}
$$

Then we define a Conflict Zone (yellow zone in Fig. 4), $C$, as the region in which the UAV feels the threat of the object, yet does not consider immediate action necessary until the estimated time to collision (TTC) between them is below a predefined threshold.

$$
\boldsymbol{C}=\left\{\gamma_{3} \leq\left\|\boldsymbol{P}_{Q}-\boldsymbol{P}_{p_{i}}\right\|-\lambda_{i} \leq \gamma_{2}, i \in \mathbb{Z}\right\}
$$

Lastly, Detection Zone, $\boldsymbol{D}$, as an exterior zone around the UAV is defined, as shown in green in Fig. 4. The UAV merely feels the presence of objects, and tries to establish tracking and velocity estimation of the objects in this region, in case they come closer and violate zone of another safetylevel. Beyond this zone, objects are considered non-existent to the UAV since they are beyond the detection range of the LIDAR sensor.

$$
\boldsymbol{D}=\left\{\gamma_{2} \leq\left\|\boldsymbol{P}_{Q}-\boldsymbol{P}_{p_{i}}\right\|-\lambda_{i} \leq \gamma_{1}, i \in \mathbb{Z}\right\}
$$

\section{B. Collision Avoidance}

In this subsection, we introduce our proposed collision avoidance controller, which generates collision avoidance control commands for lower-level vehicle controllers. This controller design is inspired by theory from missile guidance.

Fig. 5 depicts the engagement geometry between UAV and one object (represented as a circle), where $\sigma$ is the line-ofsight angle and $R$ is the Euclidean distance between them. In a classical missile guidance problem, their relative kinematics is described by following two differential equations:

$$
\begin{gathered}
\dot{R}=v_{p_{x}} \cos \sigma+v_{p_{y}} \sin \sigma-v_{Q_{x}} \cos \sigma-v_{Q_{y}} \sin \sigma \\
\dot{\sigma}=\frac{1}{R}\left(v_{p_{y}} \cos \sigma-v_{p_{x}} \sin \sigma-v_{Q_{y}} \cos \sigma+v_{Q_{x}} \sin \sigma\right)
\end{gathered}
$$

where $\boldsymbol{v}_{Q}=\left(v_{Q_{x}}, v_{Q_{y}}\right)$ and $\boldsymbol{v}_{p}=\left(v_{p_{x}}, v_{p_{y}}\right)$ represent 2D velocities of the UAV and object repsectively.

It is well known in missile guidance [13] that two vehicles are on a collision course, if following conditions are satisfied:

$$
\dot{R}<0 \quad \text { and } \quad \dot{\sigma}=0
$$

The intuition behind our avoidance strategy is that since we know the conditions for two entities being on a collision course, we could then control the UAV in such a way that they are constantly on a non-collision course by taking the reverse of these conditions, which are

$$
\dot{R} \geq 0 \quad \text { and } \quad \dot{\sigma} \neq 0
$$




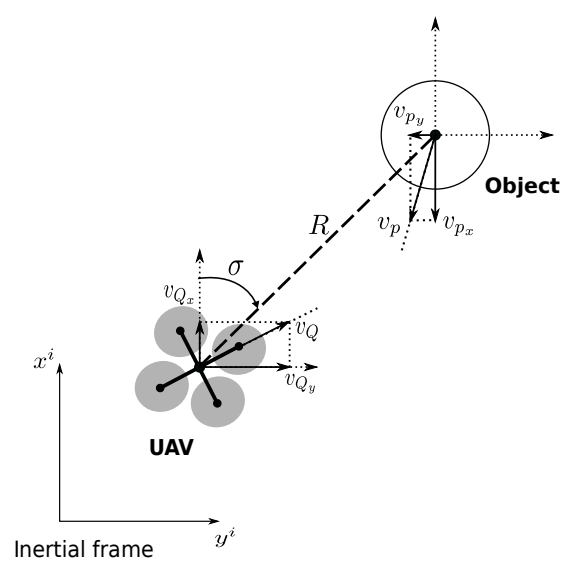

Fig. 5. Engagement Geometry of UAV and an Object (top-down view)

With above considerations in mind, we formulate the avoidance control problem as follows:

$$
\begin{array}{ll}
\max _{r, \psi} & \sum_{i=1}^{n} \zeta_{i}\left|\dot{\sigma}_{i}(r, \psi)\right|+\sum_{i=1}^{n} \eta_{i} \dot{R}_{i}(r, \psi) \\
\text { s.t. } & 0 \leq r \leq r_{\max } \\
& -\frac{\pi}{2}+\psi_{0} \leq \psi \leq \frac{\pi}{2}+\psi_{0} \\
& \zeta_{i}>0, \eta_{i}>0 \quad \forall i \in \mathbb{Z}
\end{array}
$$

where

$$
\begin{array}{r}
r=\left\|\boldsymbol{v}_{Q}\right\| \\
v_{Q_{x}}=r \cos \psi \\
v_{Q_{y}}=r \sin \psi
\end{array}
$$

and $\boldsymbol{v}_{Q}=\left(v_{Q_{x}}, v_{Q_{y}}\right)$ represent $2 \mathrm{D}$ velocity of the UAV, $\psi_{0}$ is the initial yaw angle of the UAV, $r_{\max }$ is the magnitude of maximum desirable velocity of the UAV (it is set to $1.5 \mathrm{~m} / \mathrm{s}$ in the simulation).

In this formulation, we have chosen $r$ and $\psi$ as decision variables instead of $v_{Q_{x}}, v_{Q_{y}}$ because in this way we simplify the optimization problem by avoiding nonlinear constraints.

\section{Simulation AND Results}

We conducted simulation with proposed vechicle controller, emergency evaluation policy and avoidance strategy using Matlab/Simulink. The simulation scenario from topdown view is as follows: The UAV flies towards east from location $(0,0) \mathrm{m}$ with initial velocity $(1,0) \mathrm{m} / \mathrm{s}$, while two objects fly towards the UAV with constant velocities: Object 1 (noted as $o_{1}$ ) from position $(30,-10) m$ with velocity $(-3,1) \mathrm{m} / \mathrm{s}$, object 2 (noted as $\left.o_{2}\right)$ from position $(30,30) \mathrm{m}$ with velocity $(-2,-1) \mathrm{m} / \mathrm{s}$. The UAV has an anti-object zone with radius $\gamma_{4}=1.5 \mathrm{~m}$, while object 1 and object 2 have minimum safety region with radii $\lambda_{1}=1 \mathrm{~m}$ and $\lambda_{2}=2 \mathrm{~m}$ respectively, all of which are indicated as red circles in the Fig. 6. We also set $\gamma_{3}=10 m+\gamma_{4}, \gamma_{2}=20 m+\gamma_{4}$ and $\gamma_{1}=30 \mathrm{~m}$. Several salient moments of the simulated flight are captured, as shown in Fig. 6.

In subfigure at time $t=0.000 \mathrm{~s}$, the green arrows represent initial velocities, the sizes of the arrows are in proportion
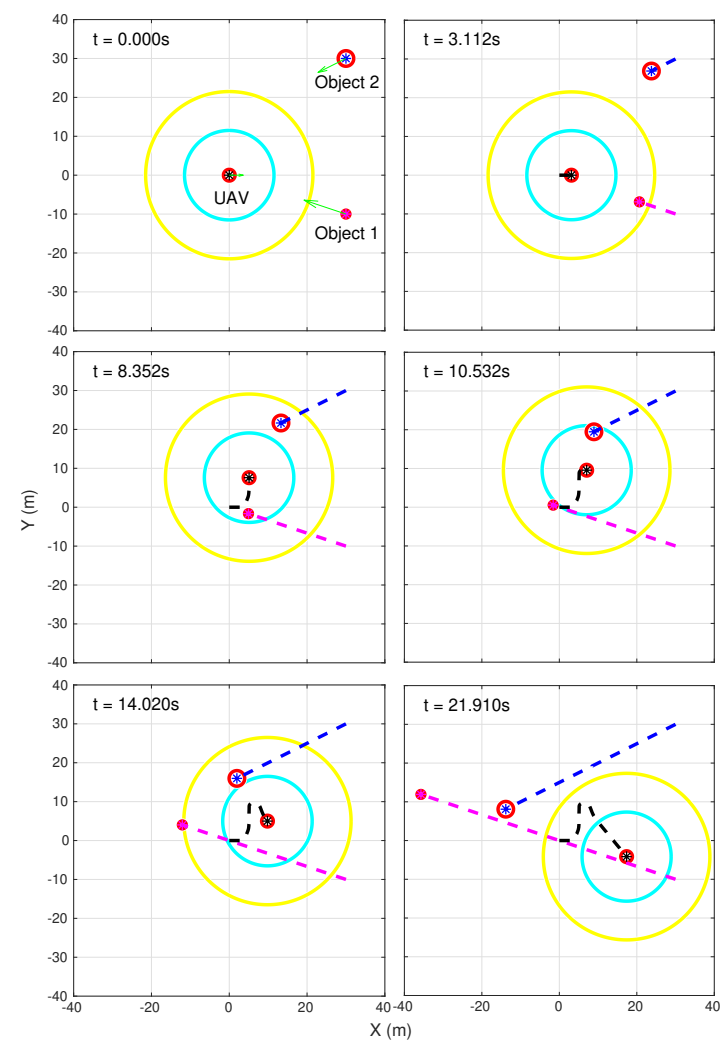

Fig. 6. Simulated Flight from top-down view: thick dashed lines represent trajectories (black for UAV, magenta for object 1, blue for object 2), yellow and cyan circles around UAV visualize the exterior borders of conflict zone and avoidance zone, red circles around the UAV and both objects visualize the minimum safety zone

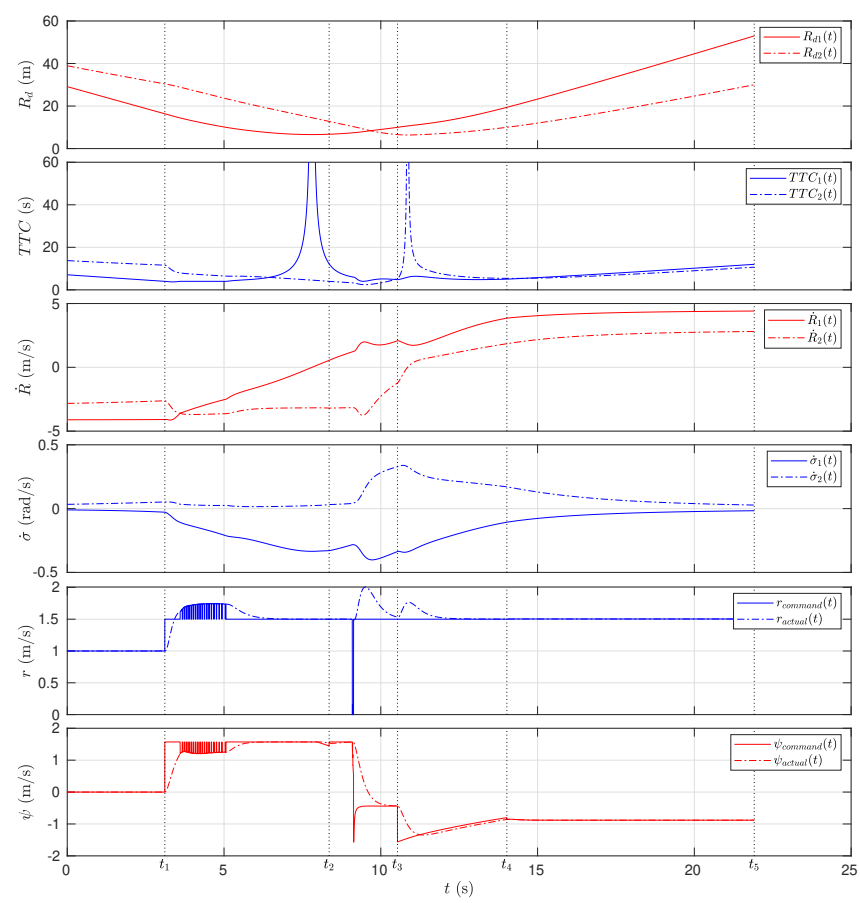

Fig. 7. Simulation Result Analysis: $t_{1}=3.112 \mathrm{~s}, t_{2}=8.362 \mathrm{~s}, t_{3}=$ $10.532 \mathrm{~s}, t_{4}=14.020 \mathrm{~s}, t_{5}=21.910 \mathrm{~s}$ 
to the magnitude of the velocities. The yellow and cyan circles around UAV visualize exterior borders of the conflict zone and avoidance zone respectively. Initially both objects are outside the conflict zone of the UAV. Subfigure at time $t=3.112 \mathrm{~s}$ depicts the moment that $o_{1}$ is in the conflict zone of the UAV, and the UAV starts to deem $o_{1}$ as imminent threat as the their estimated time to collsion $T T C_{1}$ now goes just below the predefined threshold (the threshold for TTC is set to $4 s$ in this simulation). Emergency avoidance behvior of the UAV is thus triggered. At time $t=8.352 \mathrm{~s}$, both objects pose imminent threat to the UAV, and thus influence its avoidance maneuver, as $o_{1}$ is now in zone $\boldsymbol{A}$ of the UAV while $o_{2}$ is in zone $\boldsymbol{C}$ and $T T C_{2}$ is just below the predefined threshold. At time $t=10.532 \mathrm{~s}, o_{1}$ leaves zone $\boldsymbol{A}$ of the UAV, while $o_{2}$ is within the zone $\boldsymbol{A}$. Since $T T C_{1}$ is above threshold, at this point in time only $O_{2}$ is considered as imminent threat and causes the UAV to take avoidance action. At time $t=14.020 \mathrm{~s}$, both objects stop posing threat to the UAV, as $o_{1}$ is in the conflict zone, and $o_{2}$ just goes out of the avoidance zone, and both $T T C_{1}$ and $T T C_{2}$ are above the threshold. From this moment on, the UAV stops taking avoidance action, and continues to fly in current velocity. At time $t=21.910$, both objects are outside zone $\boldsymbol{D}$ of the UAV, which means that at this moment the UAV no longer detects any object, it can now gradually come to a stop or continue flight depending on the predefined preference of the human pilot while signaling to the pilot that the control of the UAV is now returned.

In order to obtain a more qualitative view of the simulated flight, we present following relevant quantities over time in Fig. 7. Particularly, in order to take into account sizes of the UAV and the objects instead of considering them as point mass, we use $R_{d i}$ instead of $R_{i}$ to represent the distance between an object and the UAV, where $R_{d i}=$ $R_{i}-\gamma_{4}-\lambda_{i}, i \in \mathbb{Z}$. From the last two subfigures of Fig. 7 we can see that with our proposed vehicle controller the UAV is in general following commands quite well. We notice that in the $r(t)$ subfigure, despite that we set the maximum desirable velocity of the UAV to $1.5 \mathrm{~m} / \mathrm{s}$, the velocity still temporarily goes up to $2 \mathrm{~m} / \mathrm{s}$. We also notice that this velocity overshoot happens particularly when the UAV is turning (yawing). It is mainly caused by the undesired rolling while yawing, since they are coupled in our UAV model, which also reflects real-world situation. From the first two subfigures of Fig. 7, we can understand why and when the avoidance behavior of UAV is invoked from a qualitative perspective. The two sufigures in the middle visualize the evolution of $\dot{R}$ and $\dot{\sigma}$ over time, and how they are influenced by the avoidance behavior of UAV during certain period of time.

After evaluation of the overall simulation results, we can see that with the proposed vehicle controller, emergency evaluation policy and collision avoidance strategy, the UAV is capable of avoiding multiple moving objects at the same time.

\section{CONCLUSION AND Future Work}

In this paper we present a complete 2D LIDAR-based sense-and-avoid solution for assisting unskilled pilot in ensuring safe flight. Within this overall solution framework, we propose a novel nonlinear vehicle control system adapted to the characteristics of LIDAR sensing, a heuristic emergency evaluation policy and a novel avoidance control strategy which takes inspiration from missile guidance. The simulation results suggest that the proposed sense-and-avoid solution is capable of handling complex environments where multiple moving objects are present, and thus ensure safe flight of the UAV.

In the future we plan to investigate other reactive avoidance algorithms and compare with the one proposed in this paper. Furthermore, in this paper we conceptually present a complete sense-and-avoid solution, while in practice we still need to implement the proposed components in $\mathrm{C}++$ and integrate with the sensing module from our previous work [11], or from an improved version.

\section{REFERENCES}

[1] I. Mahjri, A. Dhraief, and A. Belghith, "A review on collision avoidance systems for unmanned aerial vehicles," in International Workshop on Communication Technologies for Vehicles. Springer, 2015, pp. 203-214.

[2] J. Kuchar, J. Andrews, A. Drumm, T. Hall, V. Heinz, S. Thompson, and J. Welch, "A safety analysis process for the traffic alert and collision avoidance system (tcas) and see-and-avoid systems on remotely piloted vehicles," in AIAA 3rd" Unmanned Unlimited" Technical Conference, Workshop and Exhibit, 2004, p. 6423.

[3] T. Marinho, M. Amrouche, V. Cichella, D. Stipanovi, and N. Hovakimyan, "Guaranteed collision avoidance based on line-of-sight angle and time-to-collision," in 2018 Annual American Control Conference (ACC), June 2018, pp. 4305-4310.

[4] S. Hrabar, "Reactive obstacle avoidance for rotorcraft uavs," in 2011 IEEE/RSJ International Conference on Intelligent Robots and Systems. IEEE, 2011, pp. 4967-4974.

[5] R. Sharma, J. B. Saunders, and R. W. Beard, "Reactive path planning for micro air vehicles using bearing-only measurements," Journal of Intelligent \& Robotic Systems, vol. 65, no. 1-4, pp. 409-416, 2012.

[6] J. Israelsen, M. Beall, D. Bareiss, D. Stuart, E. Keeney, and J. van den Berg, "Automatic collision avoidance for manually tele-operated unmanned aerial vehicles," in 2014 IEEE International Conference on Robotics and Automation (ICRA). IEEE, 2014, pp. 6638-6643.

[7] S. Bouabdallah and R. Siegwart, "Design and control of a miniature quadrotor," in Advances in unmanned aerial vehicles. Springer, 2007, pp. $171-210$.

[8] S. A. Raza and W. Gueaieb, "Intelligent flight control of an autonomous quadrotor," in Motion Control. IntechOpen, 2010.

[9] P. Corke, Robotics, vision and control: fundamental algorithms in MATLAB® second, completely revised. Springer, 2017, vol. 118.

[10] H. Voos, "Nonlinear control of a quadrotor micro-uav using feedbacklinearization," in 2009 IEEE International Conference on Mechatronics. IEEE, 2009, pp. 1-6.

[11] M. Wang, H. Voos, and D. Su, "Robust online obstacle detection and tracking for collision-free navigation of multirotor uavs in complex environments," in 2018 15th International Conference on Control, Automation, Robotics and Vision (ICARCV). IEEE, 2018, pp. 12281234.

[12] E. J. Rodríguez-Seda, D. M. Stipanović, and M. W. Spong, "Collision avoidance control with sensing uncertainties," in Proceedings of the 2011 American Control Conference. IEEE, 2011, pp. 3363-3368.

[13] P. Zarchan, Tactical and strategic missile guidance. American Institute of Aeronautics and Astronautics, Inc., 2012. 